\title{
A comparison in terms of resilience and anxiety between nurses working in COVID-19 wards and nurses working in other wards: a descriptive cross-sectional study in southern Iran
}

\author{
Soghra Karamizade¹, Mostafa Bijani², Azizallah Dehghan³, Zhila Fereidouni² \\ 'Student Research Committee, Fasa University of Medical Sciences, Fasa, Iran \\ ${ }^{2}$ Department of Medical Surgical Nursing, Fasa University of Medical Sciences, Fasa, Iran \\ ${ }^{3}$ Noncommunicable Diseases Research Center, Fasa University of Medical Sciences, Fasa, Iran
}

Neuropsychiatria i Neuropsychologia 2021; 16, 3-4: 124-130

Address for correspondence:

Dr Mostafa Bijani, Assist. Prof.

Department of Medical Surgical Nursing

Fasa University of Medical Sciences

Fasa, Iran

e-mail: bizhani_mostafa@yahoo.com

\begin{abstract}
Introduction: Nurses as health defenders in the front line of COVID-19 are among the high-risk and vulnerable groups for this disease. Moreover, they experience a lot of anxiety as well as psychological and emotional strain. In such situations, it is extremely important to pay attention to their resilience. Therefore, the present study attempted to compare resilience and anxiety between nurses working in the COVID-19 wards and nurses working in other wards in southern Iran in 2020.

Material and methods: This descriptive cross-sectional study was conducted by complete enumeration, meaning that the sampling process was done in a full-census manner. For the purpose of this study, 1857 nurses (866 nurses working in the COVID-19 wards and 991 nurses working in other wards) were included. To collect the required data, this study applied demographic surveys, the Corona Disease Anxiety Scale (CDAS), and the Connor-Davidson Resilience Scale (CD-RISC).

Results: The mean and standard deviation of the anxiety and resilience scores of nurses working in the COVID-19 wards were $44.11 \pm 7.48$ and $73.09 \pm 15.76$, respectively. Moreover, the anxiety and resilience scores of nurses working in other wards were $41.57 \pm 6.70$ and $75.58 \pm 8.34$, respectively. Also, a statistically significant correlation was found between resilience and anxiety $(r=-0.47, p<0.001)$.

Conclusions: This study showed that nurses are dealing with high levels of anxiety during the COVID-19 pandemic. It was concluded that due to the effect of resilience on the reduction of anxiety, nurse managers must apply the necessary psychological skill programs, in order to enhance psychological capabilities, improve nurses' resilience, and reduce coronavirus anxiety.
\end{abstract}

Key words: COVID-19, anxiety, resilience, nurses.

\section{Introduction}

Nurses face various types of stress due to the nature of their occupation; for instance, they are always exposed to highly contagious and deadly infectious diseases, including the novel coronavirus infection (COVID-19), which can consequently cause physiological and psychological destructive effects such as stress and anxiety (Wang et al. 2020). COVID-19 was first identified in China in 2019, then rapidly spread to all parts of the world. Therefore, this outbreak was announced as a pandemic by the World Health Organization (Lai et al. 2020). In January 2020, the World Health Organization identified the coronavirus epidemic as a public health emergency of international concern (PHEIC) (Santarone et al. 2020). This organization also reported that nurses as health defenders in the front line of COVID-19 are among the highrisk groups vulnerable to coronavirus. Moreover, they consequently experience a lot of anxiety as well as psychological and emotional strain (Legido-Quigley et al. 2020). Roy et al. (2020) reported that nurses are mostly suffering from stress, anxiety, depression, and post-traumatic stress disorder (PTSD) during the COVID-19 pandemic. Accordingly, it is necessary for healthcare executives and the Emergency Management Committee to pay special attentions to the psychological health status, levels of anxiety 
and depression, and occupational burnout. Under critical and complex conditions such as the COVID-19 pandemic, it is essential to pay more attention to the individual and psychological capacities, which could help a person in resisting, surviving, and even improving his/her personality traits in such difficulties (Motie et al. 2021). One of the most influential human capabilities, which helps people to be effectively adapted to stressful conditions as well as psychological and emotional strains and consequently increases their resistance to problems, is resilience (Cooke et al. 2016).

Resilience is defined as a person's capability to resist problems or to return to his/her normal status after facing a stressful situation (Ang et al. 2018; Scoloveno et al. 2016). The study by Liu et al. (2020) showed that nurses mostly experience high stress and anxiety due to a lack of high resilience and psychological capabilities in taking care of patients in the current COVID-19 crisis. Moreover, Jackson (2018) stated that nurses should particularly promote their workplace resilience especially under critical and tough conditions at work so that they can overcome their negative experiences and then convert them into positive ones. Actually, resilience corrects or modifies the adverse effects of unfavorable conditions at work, increases the psychological health statuses of medical staff, and improves the nurses' quality of care. Otherwise, nurses will experience poor working conditions, which can subsequently damage them psychologically. Accordingly, it is very difficult to show professional skills and provide high-quality professional healthcare services in such an environment and working conditions.

\section{Background in Iran}

The pattern of spread of COVID-19 has been complex in Iran. Currently, Iran is faced with the fourth wave of the pandemic, while many other countries are going through the second wave. Although the majority of Iranian people wear masks, the country's inappropriate economic situation has put very few restrictions on work, activity, and travel in all cities; therefore, a large number of people use public transportation every day, and the streets are crowded. In addition, all employees are at work every day and the rate of telecommuting from home is very low. These factors have led about 2 million Iranian people to become infected with COVID-19, of whom over 65,000 have lost their lives. During the COVID-19 pandemic, more than 100,000 nurses in Iranian hospitals became infected with the coronavirus. Iranian nurses, similar to nurses working in other countries, have experienced high job anxiety and stress in the current COVID-19 crisis.

Due to the high spread of COVID-19 and the consequent involvement of medical staff, especially nurses who are known as health defenders in the front line of COVID-19, it was necessary to use the results of the previous studies conducted on the above-mentioned issues; however, no study was found on the comparison of resilience and anxiety between nurses working in the COVID-19 wards and nurses working in other wards in Iran. Therefore, the present study was designed to compare resilience and anxiety in nurses working in the COVID-19 wards and nurses working in other wards in southern Iran in 2020.

\section{Material and methods}

This was a descriptive cross-sectional study. The statistical population of this research included the nurses working in teaching hospitals affiliated to Shiraz, Fasa, Jahrom, Darab, and Larestan Universities of Medical Sciences in southern Iran. This study was conducted using convenience sampling. Nurses who met the inclusion criteria were then invited to participate in the study. Finally, 1857 nurses (866 nurses working in the COVID-19 wards and 991 nurses working in other wards) were enrolled in the current study.

The needed data were gathered within four months from September to December 2020. The inclusion criteria were being willing to participate, having at least one year's work experience and not having any severe physical or mental health problems. Subjects who failed to answer over half of the questions on the questionnaires and did not return the questionnaires were excluded.

In the present study, it was attempted to fill out the questionnaires via phone call, email, and in some cases in person in terms of the health protocols to control the spread of this infectious disease. Demographic surveys (including different items such as age, gender, educational level, work experience, and marital status), the Corona Disease Anxiety Scale, and a resilience questionnaire were used to gather data.

The Corona Disease Anxiety Scale was prepared and then validated to measure anxiety caused by coronavirus in Iran. Correspondingly, the final version of the present questionnaire 
consists of 18 items which address two dimensions: items 1 to 9 address psychological factors and items 10 to 18 assess physical factors, which were ranked on a four-point Likert scale (never $=0$, sometimes $=1$, usually $=2$, and always $=3$ ). Therefore, the highest and lowest scores that can be obtained by the participants were 0 and 54, respectively. Scores ranging from 0 to 16 show no anxiety or mild anxiety, scores ranging from 17 to 29 show moderate anxiety, and scores ranging from 30 to 54 show severe anxiety. The Cronbach's alphas of the first and second dimensions were found to be 0.87 and 0.86 respectively. The Cronbach's $\alpha$ of the entire instrument was found to equal 0.91. (Alipour et al. 2020). Another study in Iran reported the internal homogeneity of the questionnaire to equal a Cronbach's $\alpha$ of 0.89 (Amirfakhraei et al. 2020).

The Connor-Davidson Resilience Scale has 25 questions scored from zero (never) to four (always). To obtain the total score on this scale, all scores should be added up. The final score ranges from 0 to 100 . Scores ranging from 0 to 33 indicate poor resilience, scores ranging from 34 to 67 indicate moderate resilience, and scores greater than 68 indicate good resilience (Connor and Davidson 2003).

Mohammadi et al. (2006) translated this questionnaire and also validated it. To assess the reliability of the scale, the researchers measured its Cronbach's $\alpha$. To assess the validity of the scale, the researchers used the confirmatory factor analysis method - the results showed that the instrument was a one-factor scale. The Cronbach's $\alpha$ was found to be 0.93 . Similarly, Bigdeli et al. (2013) confirmed that the instrument is reliable with a Cronbach's $\alpha$ of 0.9.

\section{Ethical considerations}

All participants gave written informed consent to participate in the study. The present study was conducted in accordance with the principles of the revised Declaration of Helsinki, a statement of ethical principles which guides physicians and other participants in medical research involving human subjects. The participants were assured of their anonymity and confidentiality of their information. Moreover, the study was approved by the local Ethics Committee of Fasa University of Medical Sciences, Fasa, Iran (Ethical code: IR.FUMS.REC.1399.096).

Statistical analyses were performed using the independent $t$-test, Mann-Whitney $U$ test, linear regression, $\chi^{2}$ test, Kruskal-Wallis test, and Spearman's correlation coefficient in SPSS software (version 22.0). Also, the $p$-value less than $0.05(p \leq 0.05)$ was considered as statistically significant.

\section{Results}

Out of 1857 nurses who participated in this study, 866 were working in the COVID-19 ward and 991 nurses were working in other wards. Table 1 illustrates other demographic characteristics of the participants.

The results showed that the mean and standard deviation of the anxiety and resilience scores of the nurses working in the COVID-19 wards were $44.11 \pm 7.48$ and $73.09 \pm 15.76$, respectively. Furthermore, the anxiety and resilience scores of the nurses working in other wards were obtained as $41.57 \pm 6.70$ and 75.58 \pm 8.34 , respectively. Additionally, a significant relationship was observed between age and work experience in the nurses working in the

Table 1. Frequency distribution of demographic characteristics of the subjects in terms of gender, marital status, and educational level

\begin{tabular}{|c|c|c|c|c|c|}
\hline \multirow[t]{2}{*}{ Variables } & \multicolumn{2}{|c|}{ COVID-19 ward } & \multicolumn{2}{|c|}{ Other wards } & \multirow[t]{2}{*}{$P$-value } \\
\hline & $n$ & $\%$ & $n$ & $\%$ & \\
\hline \multicolumn{6}{|l|}{ Gender } \\
\hline Male & 255 & 29.4 & 536 & 54.1 & \multirow[t]{2}{*}{$<0.001$} \\
\hline Female & 611 & 70.6 & 455 & 45.9 & \\
\hline \multicolumn{6}{|l|}{ Marital status } \\
\hline Single & 356 & 41.1 & 455 & 45.9 & \multirow[t]{2}{*}{0.039} \\
\hline Married & 510 & 58.9 & 536 & 54.1 & \\
\hline \multicolumn{6}{|l|}{ Level of education } \\
\hline Associate degree & 42 & 4.8 & 3 & 0.3 & \multirow[t]{4}{*}{$<0.001$} \\
\hline Bachelor of Science & 748 & 86.4 & 925 & 93.3 & \\
\hline Master of Science & 76 & 8.8 & 48 & 4.8 & \\
\hline Ph.D. & 0 & 0 & 15 & 1.5 & \\
\hline
\end{tabular}


COVID-19 ward and other wards and their levels of anxiety and resilience (Table 2).

Spearman's rank correlation coefficient showed a significant relationship between resilience and anxiety. In this regard, as resilience score increases, patients' anxiety decreases $(p<0.001, r=-0.47)$. Spearman's rank correlation coefficient also showed a significant relationship of age and work experience with anxiety and resilience levels (Table 3).

In the present study, the findings showed a significant relationship between gender and the nurses' anxiety and resilience, in such a way that the male nurses showed lesser anxiety and higher resilience compared to the female nurses. In addition, no significant relationship was found between these levels and degree of education (Table 4).

The results of the linear regression test showed that there was a significant correlation between gender, level of education and ward on the one hand and resilience and anxiety on the other (Table 5).

\section{Discussion}

COVID-19 disease is a life-threatening disease that has frightened all people worldwide. Its high rates of incidence and mortality all over the world have led humans to experience lots of psychological and emotional strain. Moreover, this disease has deeply affected people's lives and social activities and consequently their mental and psychological security (Cai et al. 2020; Habibzadeh et al. 2020), especially those of the treatment team and nurses in particular. This is because they are in direct physical contact with patients with COVID-19. It is clear that nurses experience high levels of anxiety and stress when providing care for these patients (Xiang et al. 2020).

Table 2. Relationship among age and work experience of nurses working in the COVID-19 ward and nurses working in other wards and their levels of anxiety and resilience

\begin{tabular}{|c|c|c|c|c|c|}
\hline \multirow[t]{2}{*}{ Variables } & \multicolumn{2}{|c|}{ COVID-19 ward } & \multicolumn{2}{|c|}{ Other wards } & \multirow[t]{2}{*}{$P$-value } \\
\hline & Mean & SD & Mean & SD & \\
\hline Age & 34.66 & 7.53 & 33.70 & 7.92 & 0.008 \\
\hline Job experience & 10.72 & 6.42 & 9.50 & 6.69 & $<0.001$ \\
\hline Anxiety & 44.11 & 7.48 & 41.57 & 6.70 & - \\
\hline Resilience & 73.09 & 15.76 & 75.58 & 8.34 & - \\
\hline
\end{tabular}

T-test and Mann-Whitney $U$ test

Table 3. Relationship among nurses' age and work experience and their anxiety and resilience levels

\begin{tabular}{lcccc} 
Variables & \multicolumn{1}{c}{ Anxiety } & \multicolumn{2}{c}{ Resilience } \\
\cline { 2 - 5 } & $\begin{array}{c}\text { Spearman's rank correlation } \\
\text { coefficient }\end{array}$ & $\begin{array}{c}\boldsymbol{P} \text {-value } \\
\text { Age }\end{array}$ & $\begin{array}{c}\text { Spearman's rank correlation } \\
\text { coefficient }\end{array}$ \\
\hline Job experience & 0.057 & 0.017 & 0.011 & 0.629 \\
\hline
\end{tabular}

Spearman's rank correlation coefficient

Table 4. Relationship among nurses' gender, education, and marital status and their anxiety and resilience levels

\begin{tabular}{|c|c|c|c|c|c|c|}
\hline \multirow[t]{2}{*}{ Variables } & \multicolumn{3}{|c|}{ Anxiety } & \multicolumn{3}{|c|}{ Resilience } \\
\hline & Mean & SD & $P$-value & Mean & SD & $P$-value \\
\hline \multicolumn{7}{|l|}{ Gender } \\
\hline Male & 44.45 & 6.67 & 0.024 & 76.02 & 11.55 & $<0.001$ \\
\hline Female & 40.54 & 7.12 & & 72.26 & 12.81 & \\
\hline \multicolumn{7}{|l|}{ Marital status } \\
\hline Single & 42.57 & 7.23 & & 73.38 & 12.30 & \\
\hline Married & 42.89 & 7.15 & & 57.23 & 12.46 & \\
\hline Level of education & & & 0.29 & & & 0.33 \\
\hline Associate degree & 41.02 & 7.85 & & 73.15 & 11.76 & \\
\hline Bachelor of Science & 43.19 & 7.23 & & 74.96 & 12.33 & \\
\hline Master of Science & 38.22 & 4.01 & & 67.54 & 12.67 & \\
\hline Ph.D. & 36.33 & 2.66 & & 75.00 & 0.000 & \\
\hline
\end{tabular}


Table 5. Relationship between variables affecting anxiety and resilience using linear regression

\begin{tabular}{lcccccc} 
Variable & \multicolumn{3}{c}{ Anxiety } & \multicolumn{3}{c}{ Resilience } \\
\cline { 2 - 7 } (Constant) & SE $\beta$ & $\beta$ & $P$-value & SE $\beta$ & $\beta$ & $P$-value \\
\hline Gender & 1.595 & 45.923 & $<0.001$ & 2.825 & 68.773 & $<0.001$ \\
\hline Marital status & 0.333 & 3.381 & $<0.001$ & 0.590 & 4.845 & $<0.001$ \\
\hline Education level & 0.324 & -0.449 & 0.166 & 0.575 & -2.224 & $<0.001$ \\
\hline Ward & 0.461 & -2.493 & $<0.001$ & 0.818 & -3.121 & $<0.001$ \\
\hline Age & 0.330 & -1.703 & $<0.001$ & 0.585 & 3.940 & $<0.001$ \\
\hline Job experience & 0.038 & 0.028 & 0.458 & 0.067 & 0.051 & 0.443 \\
\hline
\end{tabular}

The findings of this study showed that the mean score of anxiety in the nurses working in the COVID-19 ward was higher than that of the nurses working in other wards. It should be mentioned that the mean score of anxiety in the nurses working in other wards during the coronavirus pandemic was so high, as well. Mohammadi et al. (2021) in their study found that nurses caring for patients with COVID-19 experience a high level of anxiety. Furthermore, based on the participants' experiences, it was found that in those nurses who had psychological abilities; resilience could better adapt them to complex and stressful situations. In this study, Spearman's rank correlation coefficient showed a significant relationship between the levels of resilience and anxiety. In this regard, as the level of resilience increases, the level of anxiety decreases.

Odom-Forren (2020) also reported that nurses working during the COVID-19 pandemic experience higher anxiety than other medical staff, so this can adversely affect the quality of healthcare delivery, patients' safety, and nurses' quality of working life. Therefore, the health system policymakers should take the necessary measures and employ strategies to increase the resilience, psychological skills, and mental health statuses of nurses. Khanmohammadi et al. (2020) performed a study on 150 nurses working in a COVID-19 ward in northern Iran, in order to evaluate job stress and resilience. They observed that the mean score of job stress in these nurses was high. They also reported that the mean score of resilience was medium in them. Based on the findings of this study, an inverse relationship was found between resilience and job stress. In the present study, the mean scores of resilience in the nurses working in the COVID-19 wards and other wards were higher than in the aforementioned study, and this difference was due to different sample sizes and locations of study. It also shows that there was a significant relationship among work experience, age, and resilience; in other words, resilience increases along with aging and an increase in work experience, and this finding is consistent with that of the abovementioned study.

Doo et al. (2021) performed a study on 130 nurses in South Korea to compare the levels of anxiety, depression, and resilience between nurses working in a COVID-19 ward and nurses working in other wards. They observed that the mean scores of anxiety and depression were higher in these nurses compared to nurses working in other wards. They reported that the level of resilience in these nurses was medium, and also found a significant relationship between anxiety and resilience, which was consistent with the finding of the present study. This study indicated that there was significant relationship among age, resilience, and anxiety, which is consistent with the result of the present study. In another study by Roberts et al. (2021) on 255 nurses working in a COVID-19 ward in the USA to examine their levels of anxiety and resilience, it was observed that $20.9 \%$ of nurses suffered from severe anxiety. The mean score of resilience in $65 \%$ of nurses was between medium and high. Additionally, a significant relationship was observed among work experience, age, resilience, and anxiety, which was consistent with the results of this study.

In the present study, the Spearman's correlation coefficient showed that there was a significant correlation between resilience and anxiety: higher resilience scores correlated with lower anxiety in the patients. On a similar note, the results of the study of Setiawati et al. (2021) conducted in Indonesia show that the personnel who work in hospital wards assigned to COVID-19 patients experience higher levels of anxiety than the personnel in other wards do. Also, the personnel with less resilience experience greater anxiety, a finding which is consistent with the results of the present study. According to the study of Zhang et al. (2020), 
due to their lack of resilience skills, the nurses who practice in COVID-19 wards are exposed to greater occupational burnout and anxiety than other nurses are. It can be reasoned that the mental stress caused by direct contact with COVID-19 patients and the high risk of contracting COVID-19 increase nurses' anxiety over occupational injuries. Being among the top 10 countries extensively affected by COVID-19, Iran is a country where its healthcare personnel are currently dealing with the fourth wave of the infection and a fifth wave is forming in a few of its provinces. Over recent months, nurses and other members of treatment teams have been working intensively under great stress caring for COVID-19 patients. Providing safe, quality care to COVID-19 patients under the current complicated conditions entails possessing psychological capabilities, including resilience. Thus, nursing administrators must employ effective strategies, e.g. resilience training and stress management, to decrease nurses' anxiety and exposure to occupational injuries and enhance their resilience. Travers et al. (2020) reported that the psychological empowerment of nurses in COVID-19 hospital wards lowers their occupational anxiety and improves their resilience skills.

\section{Limitations}

One of the most important limitations of the present study was that some questionnaires were not completed and sent via e-mail, which may be due to the busy schedule of nurses during the coronavirus pandemic. Time limitation can be regarded as another limitation of the study. Since the variables were measured within 4 months, it is suggested that nurses' anxiety and resilience should be examined over a longer period; therefore, more accurate results on estimation of job stress and nurses' resilience can be achieved. Consequently, health system managers and policymakers can develop more comprehensive programs for the current crisis or similar crises based on these findings.

\section{Conclusions}

Nurses who care for COVID-19 patients and have to work long shifts suffer from higher levels of anxiety and are less resilient. Given the persistence of the COVID-19 crisis in the world and in Iran, it is essential that healthcare authorities take effective measures to lower nurses' anxiety and improve their resilience and psychological well-being. Nurse administrators can use the findings of the present study to provide a more appropriate work environment for nurses and to develop comprehensive plans to support nurses in the current and future crises.

\section{Acknowledgements}

The present paper was extracted from a research project registered under the ethical code (Ethical code: IR.FUMS.REC.1399.096) at Fasa University of Medical Sciences, Fasa, Iran. The authors would like to express appreciation to Fasa University of Medical Sciences and Clinical Research Development Unit of Fasa Valiasr hospital for financially supporting this research.

\section{Disclosure}

The authors declare no conflict of interest.

\section{References}

1. Alipour A, Ghadami A, Alipour Z, Abdollahzadeh H. Preliminary validation of the Corona Disease Anxiety Scale (CDAS) in the Iranian sample. Quart J Health Psychol 2020; 8: 163-175.

2. Amirfakhraei A, Masoumifard M, Esmaeilishad B, et al. Prediction of corona virus anxiety based on health concern, psychological hardiness, and positive meta-emotion in diabetic patients. J Diabetes Nurs 2020; 8: 10721083.

3. Ang SY, Uthaman T, Ayre TC. Association between demographics and resilience - a cross-sectional study among nurses in Singapore. Int Nurs Rev 2018; 65: 459-466.

4. Bigdeli I, Najafy M, Rostami M. The relation of attachment styles, emotion regulation, and resilience to well-being among students of medical sciences. Iran J Med Educ 2013; 13: 721-729.

5. Cai H, Tu B, Ma J, et al. Psychological impact and coping strategies of frontline medical staff in Hunan between January and March 2020 during the outbreak of Coronavirus Disease 2019 (COVID19) in Hubei, China. Med Sci Monit 2020; 26: e924171.

6. Connor KM, Davidson JR. Development of a new resilience scale: the Connor-Davidson resilience scale (CD-RISC). Depress Anxiety 2003; 18: 76-82.

7. Cooke FL, Cooper B, Bartram T, et al. Mapping the relationships between high-performance work systems,employee resilience and engagement: A study of the banking industry in China. Int J Hum Resour Manag 2016; 30: 1239-1260.

8. Doo EY, Kim M, Lee S. Influence of anxiety and resilience on depression among hospital nurses: A comparison of nurses working with confirmed and suspected patients in the COVID 19 and non COVID 19 units. J Clin Nurs 2021; 30: 1990-2000.

9. Habibzadeh P, Stoneman EK. The novel coronavirus: a bird's eye view. Int J Occup Environ Med 2020; 11: 65-71.

10. Jackson J. A grounded theory of the resilience process in post qualification nursing students. J Nurs Educ 2018; 57 : 371-374.

11. Khanmohammadi S, Hajibeglo S, Rashidan A, Karvan MB. Relationship of resilience with occupational stress 
among nurses in coronavirus ward of Khatam Al-Anbia Hospital, Gonbad Kavous, 2020. Neuropsychiatria i Neuropsychologia 2020; 15: 1-6.

12. Lai CC, Shih TP, Ko WC, et al. Severe acute respiratory syndrome coronavirus 2 (SARS-CoV-2) and coronavirus disease-2019 (COVID-19): The epidemic and the challenges. Int J Antimicrob Agents 2020; 55: 105924.

13. Legido-Quigley H, Mateos-García JT, Campos VR, et al. The resilience of the Spanish health system against the COVID-19 pandemic. Lancet Public Health 2020; 5: 251-252.

14. Liu Q, Luo D, Haase JE. The experiences of health-care providers during the COVID-19 crisis in China: a qualitative study. Lancet Glob Health 2020; 8: 790-798.

15. Mohammadi F, Farjam M, Gholampour Y, et al. Caregivers' perception of the caring challenges in coronavirus crisis (COVID-19): a qualitative study. BMC Nurs 2021; 20: 1-9.

16. Mohammadi $M$, Jazayeri A, Rafiei $A$, et al. Investigating the resiliency factors in subjects subjected to substance abuse. J Psychol Tabriz Univ 2006; 1: 115-130.

17. Motie M, Baluchi M, Dehnavieh R, Kalavani K. Educating nurses and improving their resilience during the Covid-19. Biomed J Sci Tech Res 2021; 32: 25374-25375.

18. Odom-Forren J. Nursing resilience in the world of COVID-19. J Perianesth Nurs 2020; 35: 555-556.

19. Roberts NJ, McAloney-Kocamana K, Lippiett K, et al. Levels of resilience, anxiety and depression in nurses working in respiratory clinical areas during the COVID pandemic. Respir Med 2021; 176: 106219.

20. Roy D, Tripathy S, Kar SK, et al. Study of knowledge, attitude, anxiety \& perceived mental healthcare need in Indian population during COVID-19 pandemic. Asian J Psychiatr 2020; 51: 102083.

21. Santarone K, McKenney M, Elkbuli A. Preserving mental health and resilience in frontline health care workers during COVID-19. Am J Emerg Med 2020; 38: 1530-1531.

22. Scoloveno R. A concept analysis of the phenomenon of resilience. J Nurs Care 2016; 5: 353.

23. Setiawati Y, Wahyuhadi J, Joestandari F, et al. Anxiety and resilience of healthcare workers during COVID-19 pandemic in Indonesia. J Multidiscip Healthc 2021; 14: 1-8.

24. Travers JL, Schroeder K, Norful AA, et al. The influence of empowered work environments on the psychological experiences of nursing assistants during COVID-19: a qualitative study. BMC Nurs 2020; 19: 98.

25. Wang D, Hu B, Hu C, et al. Clinical characteristics of 138 hospitalized patients with 2019 novel coronavirus-infected pneumonia in Wuhan, China. JAMA 2020; 323: 1061 1069.

26. Xiang YT, Yang Y, Li W, et al. Timely mental health care for the 2019 novel coronavirus outbreak is urgently needed. Lancet Psychiatry 2020; 7: 228-229.

27. Zhang Y, Wang C, Pan W, et al. Stress, burnout, and co ping strategies of frontline nurses during the COVID-19 epidemic in Wuhan and Shanghai, China. Front Psychiatry 2020; 11: 565520. 